\title{
Once Versus Twice-Daily Oral Proton Pump Inhibitor Therapy for Prevention of Peptic Ulcer Rebleeding: A Propensity Score-Matched Analysis
}

\author{
Fares Ayoub, d, Vikas Khullar ${ }^{\mathrm{b}}$, Debdeep Banerjee ${ }^{\mathrm{a}}$, Patrick Stoner ${ }^{\mathrm{a}}$, Tiffany Lambrou ${ }^{\mathrm{a}}$, \\ Donevan R. Westerveld ${ }^{\mathrm{a}}$, Wissam Hanayneh ${ }^{\mathrm{a}}$, Amir Y. Kamel ${ }^{\mathrm{c}}$, David Estores ${ }^{\mathrm{b}}$
}

\begin{abstract}
Background: After inpatient management of upper gastrointestinal bleeding (GIB) due to peptic ulcer disease (PUD), oral proton pump inhibitor (PPI) therapy is recommended at discharge to decrease rebleeding risk and improve ulcer healing. Our aim is to determine whether once-daily oral PPI dosing at hospital discharge is associated with inferior 30-day rebleeding outcomes as compared to twice-daily dosing.
\end{abstract}

Methods: We retrospectively identified 233 patients admitted with signs and symptoms of upper GIB found to be due to PUD on upper endoscopy. After inpatient management, patients discharged on once-daily oral PPI were compared to those discharged on twice-daily therapy. We utilized propensity score matching based on Rockall scores to ensure the two groups were closely matched in terms of their baseline rebleeding risk. Primary outcome was the incidence of rebleeding within 30 days. Secondary outcomes were all-cause mortality, blood transfusion requirement, requirement for interventional radiology or surgery.

Results: Overall, 49 patients were discharged on once-daily and 184 on twice-daily PPI. Recurrent bleeding occurred in 18 patients $(7.7 \%)$ within 30 days. There was no statistically significant difference in recurrent bleeding rates between once-daily $(n=7,14.3 \%)$ as compared to twice-daily PPI $(\mathrm{n}=11,6 \%)(\mathrm{P}=0.053)$. In a $1: 1$ propensity score matched analysis, there was no statistically significant difference in 30 -day recurrent bleeding rate between groups (14\% once-daily vs. $4 \%$ twice-daily, $\mathrm{P}=0.159$ ). There were no differences in secondary outcomes.

Conclusions: Once-daily oral PPI dosing at hospital discharge was

Manuscript submitted March 23, 2018, accepted April 10, 2018

aDepartment of Medicine, University of Florida, Gainesville, FL 32608, USA bDivision of Gastroenterology, Hepatology and Nutrition, Department of Medicine, University of Florida, Gainesville, FL 32608, USA

'Department of Pharmacy, University of Florida, Gainesville, FL, 32608, USA

${ }^{\mathrm{d} C}$ Corresponding Author: Fares Ayoub, Department of Medicine, University of Florida, Gainesville, 1600 SW Archer Rd., FL 32608, USA.

Email: Fares.ayoub@medicine.ufl.edu

doi: https://doi.org/10.14740/gr1011w not associated with inferior outcomes compared to twice-daily dosing in patients hospitalized for upper GIB due to PUD.

Keywords: Proton pump inhibitor; Peptic ulcer disease; Upper gastrointestinal bleeding; Non-variceal gastrointestinal bleeding; Dosing

\section{Introduction}

Peptic ulcer disease (PUD) is the most common cause of gastrointestinal bleeding (GIB) and remains a serious condition that carries a substantial risk for morbidity and mortality [1]. Rebleeding after initial stabilization is an independent predictor of mortality and is estimated to occur in $7-16 \%$ of patients [2-4]. As a result, efforts aimed at prevention of rebleeding are highly desirable as they can directly influence prognosis. Rebleeding most commonly occurs within $72 \mathrm{~h}$ of the initial bleeding episode during which intravenous (IV) therapy with a proton pump inhibitor (PPI) is often indicated $[5,6]$. The risk for rebleeding does not end at $72 \mathrm{~h}$, however, and up to $44 \%$ of patients who rebleed experience a rebleeding event after the initial $72 \mathrm{~h}$ [7]. As a result, patients are often discharged on oral PPI therapy to promote ulcer healing and prevent recurrent bleeding.

While the need for oral PPI therapy at discharge is wellestablished, the appropriate dosing is less clear. With only one prospective study [8] of 187 patients and, to our knowledge, no other prospective or retrospective studies directly addressing this question, the appropriate PPI dosing at discharge remains unclear. Despite being particularly safe in the short-term, proton pump inhibitors carry a significant economic and adverse event burden [9], thus determining appropriate dosing at the time of discharge is of particular clinical relevance.

In patients found to have GIB due to PUD, we compared 30-day rebleeding rates in those discharged on once versus twice-daily oral PPI therapy after appropriate inpatient management.

\section{Methods}

This was a retrospective cohort study conducted at the Uni- 


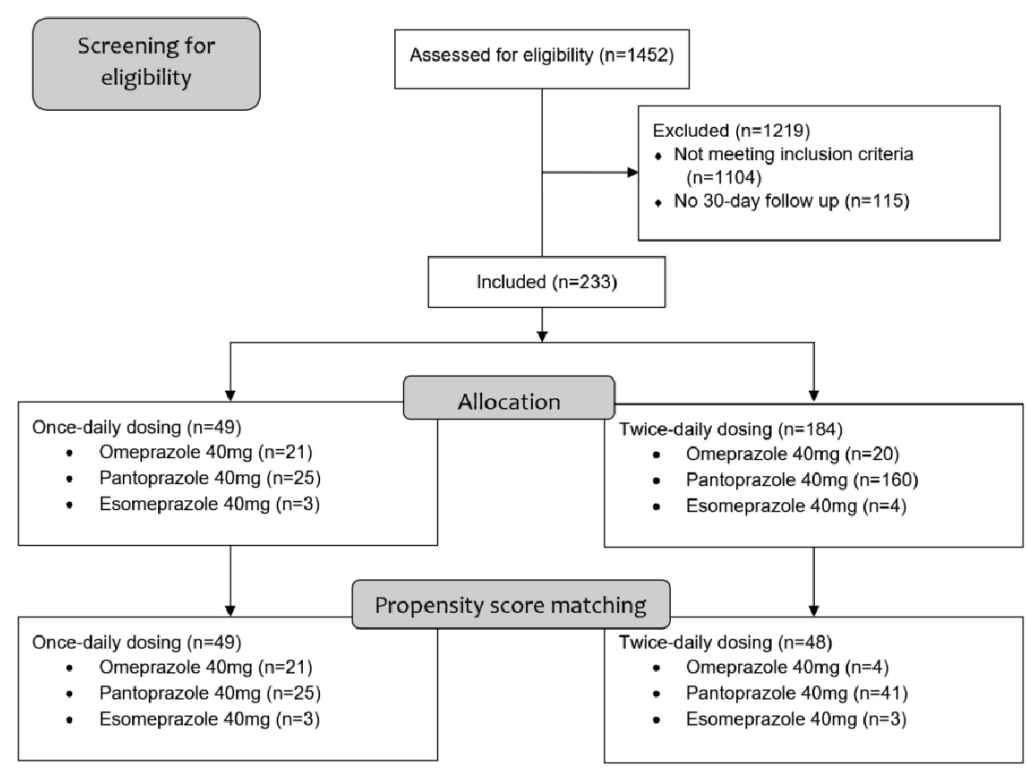

Figure 1. Flow diagram of included patients.

versity of Florida, a tertiary referral center in the southeastern United States. This study was approved by the institutional review board at the University of Florida (IRB201700669). Between March 2013 and March 2016, a prospectively maintained endoscopy database was queried to identify patients undergoing upper endoscopy for signs and symptoms of upper GIB. Patients confirmed to have upper GIB due to PUD were screened to determine whether they met inclusion/exclusion criteria. Based on the dose of oral PPI prescribed after inpatient management, patients were allocated into either the once-daily or the twice-daily dosing group. The incidence of rebleeding in each group was then recorded up to 30 days after hospital discharge.

\section{Inclusion/exclusion criteria}

Due to the retrospective design, study size was determined by the number of patients meeting inclusion/exclusion criteria. Patients aged 18 years and above presenting with signs and symptoms of upper GIB requiring hospitalization and subsequent upper endoscopy were eligible for inclusion. Inclusion was limited to those with peptic ulcers visualized on upper endoscopy deemed to be the source of bleeding by the endoscopist. Excluded were patients with a history of esophageal or gastric varices, patients without obvious source of bleeding on upper endoscopy, patients with non-peptic ulcer causes for bleeding (arteriovenous malformations, diffuse erosive gastritis, esophagitis, mechanical factors such as nasogastric tube placement), patients with a history of abnormal upper gastrointestinal anatomy due to previous gastrointestinal surgery and patients with upper gastrointestinal cancers. Patients who were lost to follow up after their initial bleeding episode were also excluded. Figure 1 shows the flow diagram of the study popu- lation.

\section{Outcomes}

The primary outcome was the incidence of rebleeding from initiation of oral therapy up to 30 days after discharge. Rebleeding was defined as recurrent hematemesis, hematochezia, melena, or blood-tinged nasogastric tube output; or recurrence of hemodynamic instability with systolic blood pressure less than $90 \mathrm{~mm} \mathrm{Hg} /$ heart rate more than $100 \mathrm{bpm}$ and a decrease in hemoglobin by more than $2 \mathrm{~g} / \mathrm{dL}$ that was attributed to upper GIB. Rebleeding episodes were confirmed by repeat upper endoscopy demonstrating a bleeding peptic ulcer. Secondary outcomes included all-cause mortality at 30 days and the following outcomes for the rebleeding episode: blood transfusion requirements, additional hospital days during rebleeding episode, requirement for interventional embolization and requirement for surgical intervention.

\section{Statistical analysis}

Univariate analysis of categorical variables was conducted using the Chi-square test or Fisher's exact test as appropriate. Parametric data was analyzed using the Student's $t$ test when two means were compared. Non-parametric data was analyzed utilizing the Mann-Whitney rank sum test when two medians were compared. All tests were two-tailed and a P-value of $<$ 0.05 was considered significant.

To account for differences in treatment group size and baseline rebleeding risk, we also conducted a secondary analysis utilizing propensity score matching. Propensity score matching can be used to reduce bias in retrospective studies, 
including selection bias and other potential confounders. Propensity score matching simulates a randomized controlled trial-like situation where the treatment and the control groups are matched in terms of selected confounders [10]. The propensity score for each subject was estimated utilizing a logistic regression model for recurrent bleeding as a function of the components of the Rockall score. The Rockall score is a validated score that predicts the risk of recurrent bleeding and mortality after upper gastrointestinal bleeding [11]. The components of the Rockall score that were utilized to generate the propensity score for each subject were age, presence of shock, comorbidities, type of lesion and the visualization of major stigmata of recent hemorrhage on upper endoscopy. After estimation of the propensity score for each subject, we performed one-to-one matching utilizing the nearest neighbor method with a caliper width of 0.2 of the standard deviation of the logit of the propensity score. By utilizing propensity score matching, subjects in both groups were matched by their baseline recurrent bleeding and mortality risk, since some of these baseline characteristics were significantly different between the two groups prior to matching. After matching, all baseline characteristics were balanced between the two groups. Statistical analysis was completed using Stata version 15 (StataCorp, College Station, TX).

\section{Results}

\section{Patients and baseline characteristics}

Of 1,452 patients screened for inclusion, 1,104 did not meet inclusion criteria. An additional 115 eligible for inclusion were excluded due to having no 30-day follow-up after their initial gastrointestinal bleeding episode. Of the remaining included 233 patients, 49 were discharged on once-daily oral PPI therapy and 184 on twice-daily oral PPI therapy (Fig. 1). Overall, mean age of participants was 61.2 years and $123(53 \%)$ were males. Median BMI was $26.7 \mathrm{~kg} / \mathrm{m}^{2}, 73(31 \%)$ patients were active smokers and 85 (37\%) reported alcohol use. Hemodynamic shock, defined as a systolic blood pressure of $<90 \mathrm{~mm}$ $\mathrm{Hg}$ with a heart rate of $>100$ beats per min, was present in 48 (20.6\%) patients at presentation. The most common presenting symptom was melena in $143(61.4 \%)$, followed by hematemesis in $46(20 \%)$, hematochezia in $30(13 \%)$ and syncope in $11(5 \%)$ patients. Thirty-four patients $(14.6 \%)$ were found to be $H$. pylori positive on biopsy after endoscopy. Prior to their bleeding episode, 118 (51\%) patients were on aspirin therapy, $101(43 \%)$ reported active non-aspirin NSAID use, $27(12 \%)$ used anti-platelet therapy, $23(10 \%)$ used warfarin, $12(5 \%)$ used direct oral anti-coagulants and $10(4 \%)$ used subcutaneous heparin. Demographics and clinical characteristics of both groups are shown in Table 1. In patients discharged on oncedaily PPI the most frequently prescribed PPI was pantoprazole $40 \mathrm{mg}(\mathrm{n}=25,51 \%)$, followed by omeprazole $(\mathrm{n}=21,43 \%)$ and esomeprazole $40 \mathrm{mg}(\mathrm{n}=3,6 \%)$. In the twice-daily group, pantoprazole $40 \mathrm{mg}$ was also the most frequently prescribed PPI $(n=160,87 \%)$, followed by omeprazole $40 \mathrm{mg}(n=20$, $11 \%)$ and esomeprazole $40 \mathrm{mg}(\mathrm{n}=4,2 \%)$. Details of dis- charge PPI types and dosing are shown in Table 2.

Before propensity score matching, significant differences were found between the two groups in gender and American Society of Anesthesiologists (ASA) score. After one-to-one propensity score matching, we identified 48 pairs of patients with baseline characteristics that were closely balanced.

\section{Endoscopic findings}

Findings on upper endoscopy after the time of initial bleeding episode are shown in Table 3. Overall, the median Rockall score was 4 in both groups. Ulcer size ranged from 2 - 40 $\mathrm{mm}$. There were $68(29.2 \%)$ patients with Forrest class ulcers $2 \mathrm{a}$ and above. The majority of ulcers were gastric $(\mathrm{n}=144$, $61.8 \%$ ) and 123 patients (52.8\%) had single ulcers on endoscopy. Prior to propensity score matching, there were significant differences in the Forrest classification of ulcers between the two groups. This was well-balanced after matching. There were no other differences between groups.

\section{Outcomes}

Overall, 18 patients (7.7\%) had endoscopically confirmed recurrent bleeding within 30 days of discharge. There was no significant difference in 30-day rebleeding rates between the two groups; seven patients $(14.3 \%)$ in the once-daily oral PPI dose group had rebleeding as compared to $11(6 \%)$ in the twice-daily oral PPI group $(\mathrm{P}=0.053)$. The lack of significant difference between the two groups persisted in the propensity score-matched analysis with seven patients (14.3\%) bleeding in the once-daily dose group as compared to two patients $(4.2 \%)$ in the twice-daily dose group $(\mathrm{P}=0.159)$. No significant differences were found between the two groups in all-cause mortality (zero in once-daily vs. four deaths in twice-daily, $\mathrm{P}=0.582$ ), blood transfusion requirement (mean 1.5 units of packed red blood cells (pRBCs) per rebleed in once-daily vs. 1.3 units in twice-daily, $\mathrm{P}=0.584$ ), additional hospital days during rebleeding episode (mean 6.7 additional days per rebleed in once-daily vs. 6.2 days in twice-daily, $\mathrm{P}=$ 0.614 ), requirement for embolization by interventional radiology or requirement for surgical intervention for the rebleeding episode (Table 4). There were four deaths in the twice-daily dosing group, three of which were due to septic shock and one due to cardiogenic shock. No deaths were directly related to a rebleeding event. To determine whether our findings persisted when patients thought to be at a low-risk of rebleeding were excluded, we conducted secondary sensitivity analyses by assessing for a change in the primary outcome after sequentially excluding patients with Rockall scores lower than 4, 5 and 6 respectively in the two groups and this did not alter our results.

\section{Discussion}

In our retrospective cohort study of 233 patients admitted for peptic ulcer bleeding, we found no significant difference in 
Table 1. Baseline Patient Characteristics in Patients Discharged on Once Versus Twice-Daily Oral PPI Therapy

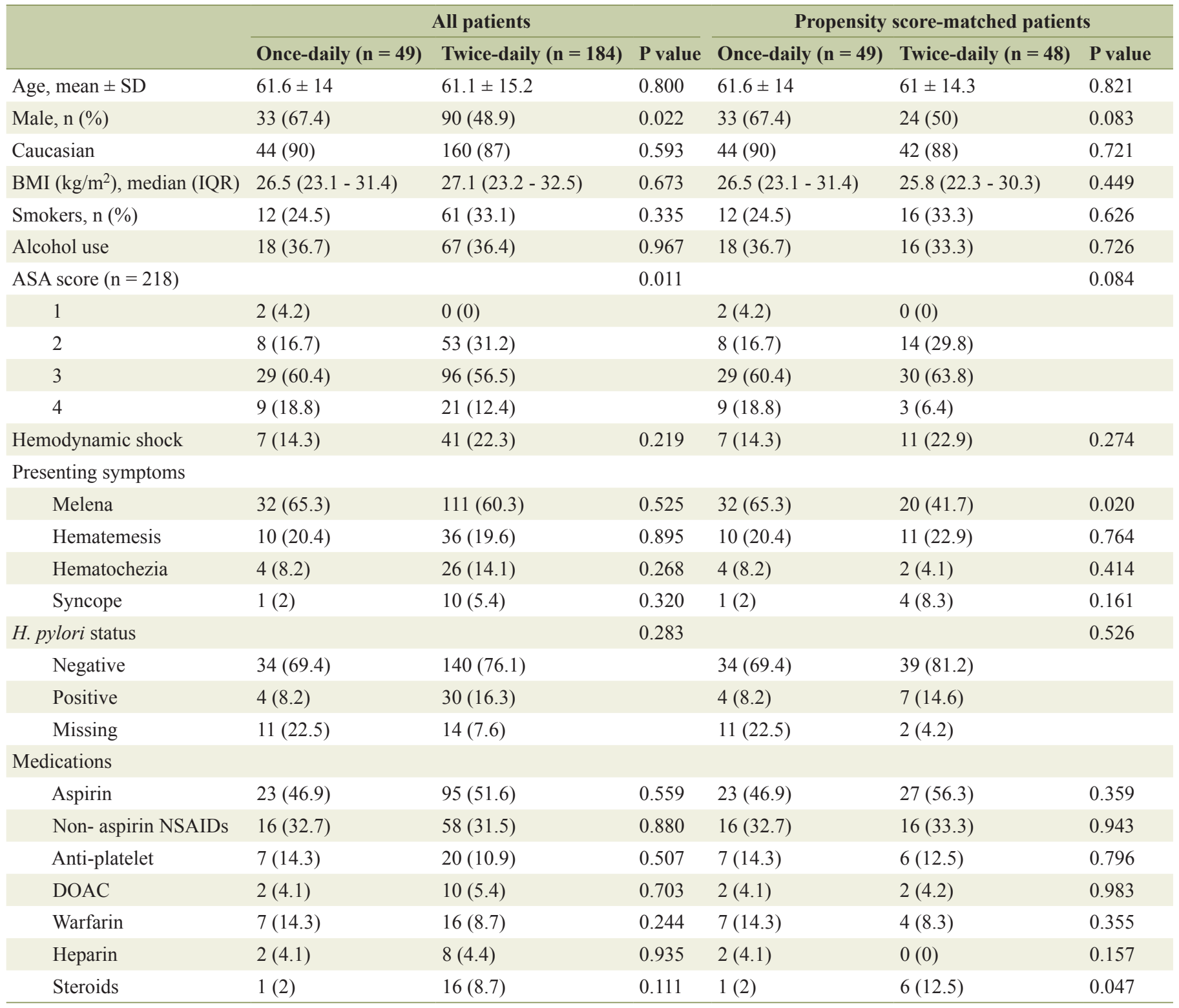

Values between parentheses are percentages unless otherwise specified. Values with a plus/minus sign $( \pm)$ represent means \pm standard deviation. BMI: body mass index; ASA: American Society of Anesthesiologists; NSAID: non-steroidal anti-inflammatory drug; DOAC: direct oral anticoagulant.

30-day rebleeding rates between patients discharged on oncedaily as compared to twice-daily oral PPI therapy. We also observed no difference in all-cause mortality, requirement for arterial embolization by interventional radiology, surgical intervention, additional hospital days or amount of packed red blood cell transfusion for rebleeding between the two groups.

Rebleeding is one of the strongest predictors of mortality in upper GIB [12]. While the advent of endoscopic and intravenous proton pump inhibitor therapy has reduced recurrent bleeding rates, it continues to occur in a substantial percentage of patients. It is well recognized that utilization of oral PPI therapy at the time of discharge reduces rebleeding rates [13]. However, the appropriate dosing of oral PPI remains unclear.
In a 2012 clinical practice guideline, the American College of Gastroenterology recommended once-daily oral PPI therapy at discharge in low-risk patients (ulcers that have flat pigmented spots or clean bases) [14]. However, no recommendation is made regarding patients deemed to be high-risk. Some providers prescribe twice-daily oral PPI therapy at discharge in an effort to improve ulcer healing and prevent rebleeding. However, evidence for such practice is scarce.

To our knowledge, only one prospective study of patients at high risk of rebleeding reported that twice-daily dosing for the first 11 days after discharge significantly decreased rebleeding risk. In their 2014 study, Cheng et al concluded that high-risk patients with Rockall score $\geq 6$ treated with oral esomeprazole 
Table 2. Details of Proton Pump Inhibitor Therapy Types, Frequency, and Dosing in the Two Groups

\begin{tabular}{llllll} 
& \multicolumn{2}{c}{ All patients } & & \multicolumn{3}{c}{ Propensity score matched patients } \\
\cline { 2 - 3 } \cline { 5 - 6 } & Once-daily $(\mathbf{n}=\mathbf{4 9})$ & Twice-daily $(\mathbf{n}=\mathbf{1 8 4})$ & & Once-daily $(\mathbf{n}=\mathbf{4 9})$ & Twice-daily $(\mathbf{n}=\mathbf{4 8})$ \\
\hline Omeprazole $40 \mathrm{mg}$ & $21(42.8 \%)$ & $20(11 \%)$ & & $21(43 \%)$ & $4(8 \%)$ \\
Pantoprazole $40 \mathrm{mg}$ & $25(51 \%)$ & $160(87 \%)$ & $25(51 \%)$ & $41(85 \%)$ \\
Esomeprazole $40 \mathrm{mg}$ & $3(6 \%)$ & $4(2 \%)$ & $3(6 \%)$ & $3(6 \%)$ \\
\hline
\end{tabular}

twice daily for 11 days after $72 \mathrm{~h}$ of intravenous esomeprazole for peptic ulcer bleeding had a significantly lower incidence of rebleeding at 1 month than those receiving once-daily oral esomeprazole (10.8\% vs. $28.7 \%)$ [8].

Our findings differ from the aforementioned study, even when we attempted to emulate their inclusion criteria in our sensitivity analysis (including only patients with a Rockall score of score $\geq 6$ ). We note that their study was conducted in a predominantly Asian population as compared to the predominantly Caucasian population in our cohort. Asians metabolize proton pump inhibitors differently due to their smaller parietal mass and have a higher prevalence of the CYP2C19 allele leading to poor metabolism and substantially higher serum concentrations of the medication $[15,16]$. Another difference in study design is their use of only oral esomeprazole as the study PPI. Esomeprazole has significant differences in its pharmacokinetics and pharmacodynamics from other PPIs which may also explain the different outcome of their study [16]. In our cohort, a limited number of patients were discharged with oral esomeprazole.

Studies utilizing gastric $\mathrm{pH}$ monitoring with PPI therapy have demonstrated a failure of daily PPI dosing to suppress acid secretion consistently over $24 \mathrm{~h}$ which may be the impetus for providers to prescribe twice-daily PPI [17]. However, while twice-daily dosing may improve the efficacy of acid suppression, studies have demonstrated periods of "acid breakthrough" despite twice-daily dosing [18]. Profound acid suppression may not always correlate with improved outcomes; in one study, suppressing gastric $\mathrm{pH}$ beyond 3.0 was not found to improve ulcer healing [19]. Interestingly, there is increas-

Table 3. Initial Endoscopic Findings in Patients Discharged on Once Versus Twice-Daily Therapy

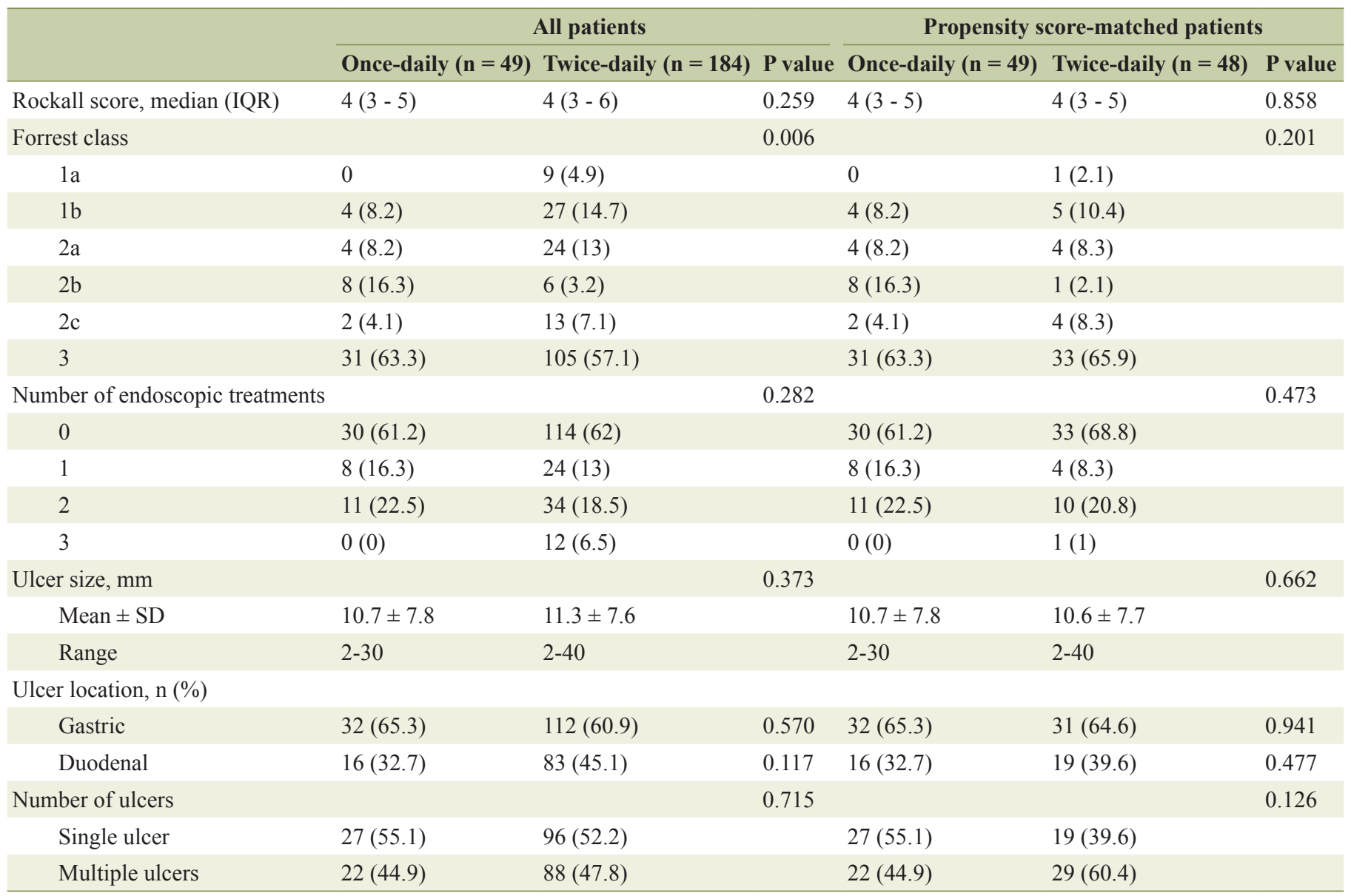




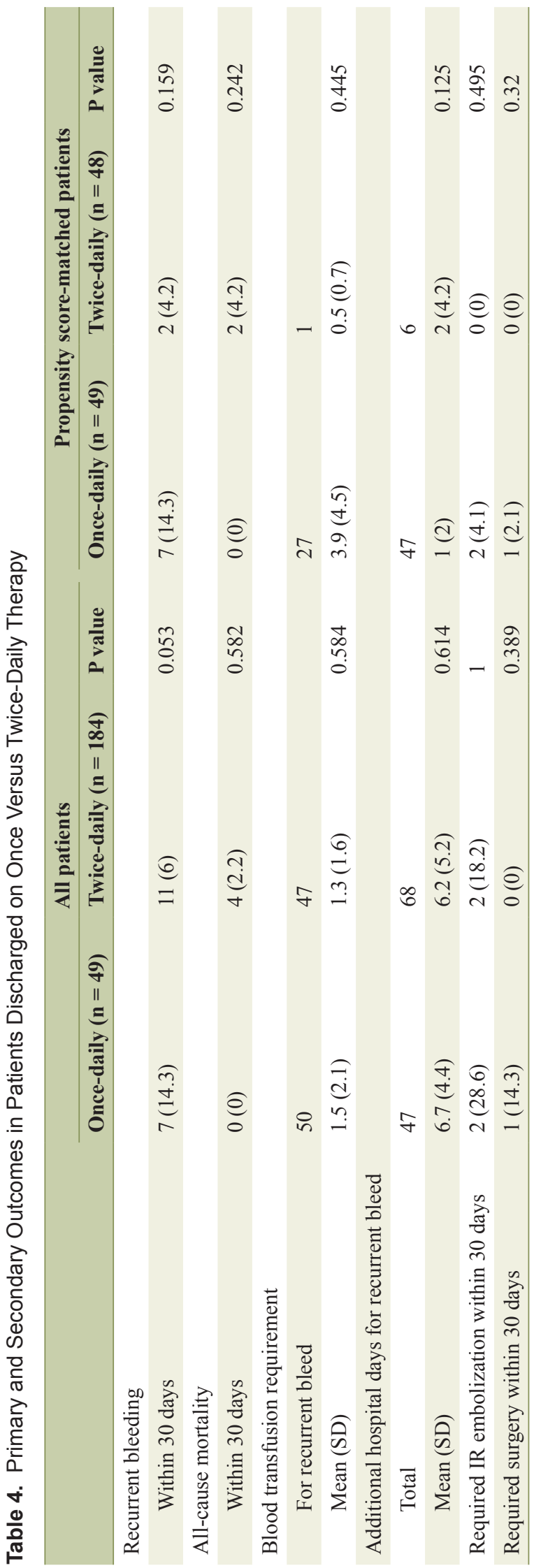

ing evidence that PPIs as a class of medication have an antiinflammatory effect that may contribute to peptic ulcer healing, independent of their acid-suppressing effects [20]. Such an anti-inflammatory effect may not necessarily increase with more frequent dosing, which may help explain our findings.

Our study adds to the very limited literature on this topic and reflects a "real-life" scenario in terms of population, setting and intervention for upper gastrointestinal bleeding. However, we do acknowledge certain limitations. Our study's retrospective design carries an inherent risk for bias and unmeasured confounders. However, we applied strict inclusion and exclusion criteria to minimize such bias leading to an $84 \%(1,219$ excluded from initial sample of 1,452 patients) reduction of patients from the initially identified sample. Due to the retrospective design, we were also unable to verify patient adherence to prescribed dosing and frequency of PPI therapy, however this is a limitation of other study designs. It is also notable that patients in our study were discharged on different types of PPIs, and there is literature to suggest subtle differences in PPI pharmacokinetics and dynamics, which may introduce some bias. However, this most closely mimics "real-life" practice where different providers prescribe different types of PPIs. Reflecting practice patterns at our institution, we had a smaller number of patients in the once-daily dose arm. However, with one-to-one propensity score matching, we attempted to very closely match these patients to those in the twice-daily dose group. Finally, the power of our study to detect a significant difference between the two groups may have been hindered by the numbers of patients included and a potential difference may exist that we were not able to detect.

In conclusion, our analysis indicates that once-daily oral PPI dosing at hospital discharge was not associated with inferior outcomes compared to twice-daily dosing in patients hospitalized for upper GIB due to PUD. We believe the risks of patient non-adherence, potential side effects, medication interactions as well as the higher financial burden associated with more frequent PPI dosing currently outweighs the potential benefits. Prospective trials in larger patient cohorts are needed to validate our findings.

\section{Grant Support}

No grants were used to support this work.

\section{Disclosures}

The authors have no financial arrangement related to the research of preparation of the manuscript.

\section{References}

1. van Leerdam ME. Epidemiology of acute upper gastrointestinal bleeding. Best Pract Res Clin Gastroenterol. 2008;22(2):209-224.

2. Barkun A, Sabbah S, Enns R, Armstrong D, Gregor J, 
Fedorak RN, Rahme E, et al. The Canadian Registry on Nonvariceal Upper Gastrointestinal Bleeding and Endoscopy (RUGBE): Endoscopic hemostasis and proton pump inhibition are associated with improved outcomes in a real-life setting. Am J Gastroenterol. 2004;99(7):12381246.

3. Vreeburg EM, Snel P, de Bruijne JW, Bartelsman JF, Rauws EA, Tytgat GN. Acute upper gastrointestinal bleeding in the Amsterdam area: incidence, diagnosis, and clinical outcome. Am J Gastroenterol. 1997;92(2):236-243.

4. Peura DA, Lanza FL, Gostout CJ, Foutch PG. The American College of Gastroenterology Bleeding Registry: preliminary findings. Am J Gastroenterol. 1997;92(6):924928.

5. Gralnek IM, Dumonceau JM, Kuipers EJ, Lanas A, Sanders DS, Kurien M, Rotondano G, et al. Diagnosis and management of nonvariceal upper gastrointestinal hemorrhage: European Society of Gastrointestinal Endoscopy (ESGE) Guideline. Endoscopy. 2015;47(10):a1-46.

6. Hwang JH, Fisher DA, Ben-Menachem T, Chandrasekhara V, Chathadi K, Decker GA, Early DS, et al. The role of endoscopy in the management of acute non-variceal upper GI bleeding. Gastrointest Endosc. 2012;75(6):11321138.

7. El Ouali S, Barkun A, Martel M, Maggio D. Timing of rebleeding in high-risk peptic ulcer bleeding after successful hemostasis: a systematic review. Can J Gastroenterol Hepatol. 2014;28(10):543-548.

8. Cheng HC, Wu CT, Chang WL, Cheng WC, Chen WY, Sheu BS. Double oral esomeprazole after a 3-day intravenous esomeprazole infusion reduces recurrent peptic ulcer bleeding in high-risk patients: a randomised controlled study. Gut. 2014;63(12):1864-1872.

9. Heidelbaugh JJ, Goldberg KL, Inadomi JM. Overutilization of proton pump inhibitors: a review of costeffectiveness and risk [corrected]. Am J Gastroenterol. 2009;104(Suppl 2):S27-32.

10. D'Agostino RB, Jr. Propensity score methods for bias re- duction in the comparison of a treatment to a non-randomized control group. Stat Med. 1998;17(19):2265-2281.

11. Rockall TA, Logan RF, Devlin HB, Northfield TC. Risk assessment after acute upper gastrointestinal haemorrhage. Gut. 1996;38(3):316-321.

12. Katschinski B, Logan R, Davies J, Faulkner G, Pearson J, Langman M. Prognostic factors in upper gastrointestinal bleeding. Dig Dis Sci. 1994;39(4):706-712.

13. Lin HJ, Lo WC, Lee FY, Perng CL, Tseng GY. A prospective randomized comparative trial showing that omeprazole prevents rebleeding in patients with bleeding peptic ulcer after successful endoscopic therapy. Arch Intern Med. 1998;158(1):54-58.

14. Laine L, Jensen DM. Management of patients with ulcer bleeding. Am J Gastroenterol. 2012;107(3):345-360; quiz 361 .

15. Furuta T, Shirai N, Sugimoto M, Ohashi K, Ishizaki T. Pharmacogenomics of proton pump inhibitors. Pharmacogenomics. 2004;5(2):181-202.

16. McColl KE, Kennerley P. Proton pump inhibitors - differences emerge in hepatic metabolism. Dig Liver Dis. 2002;34(7):461-467.

17. Tytgat GN. Shortcomings of the first-generation proton pump inhibitors. Eur J Gastroenterol Hepatol. 2001;13(Suppl 1):S29-33.

18. Peghini PL, Katz PO, Bracy NA, Castell DO. Nocturnal recovery of gastric acid secretion with twice-daily dosing of proton pump inhibitors. Am J Gastroenterol. 1998;93(5):763-767.

19. Burget DW, Chiverton SG, Hunt RH. Is there an optimal degree of acid suppression for healing of duodenal ulcers? A model of the relationship between ulcer healing and acid suppression. Gastroenterology. 1990;99(2):345351.

20. Kedika RR, Souza RF, Spechler SJ. Potential anti-inflammatory effects of proton pump inhibitors: a review and discussion of the clinical implications. Dig Dis Sci. 2009;54(11):2312-2317. 